\title{
MATHEMATICAL MODELLING OF RETINAL TEAR FORMATION: IMPLICATIONS FOR THE USE OF HEAVY LIQUIDS
}

\author{
J. D. STEVENS, ${ }^{*}$ I. L. JONES, $†$ M. WARNER, $†$ M. J. LAVIN, $\ddagger$ P. K. LEAVER* \\ London, Cambridge and Manchester
}

\begin{abstract}
SUMMARY
When force is applied to detached retina it elevates, stretches, and becomes irreversibly deformed. Finally it tears, and the timing and location of tear formation are determined by the distribution of stress within the retina. This stress distribution is dependent upon the retinal contour. We have tested this using retinas of freshly enucleated bovine eyes. A suture was attached to isolated retinal tissue using butylacrylate (Histoacryl) glue and traction was applied to this suture so stretching the retina. We present a mathematical model of the in vitro data obtained, with predictions for local retinal internal tensions. We show that these are altered by the addition of hydrostatic forces due to the presence of heavy liquids. These findings have implications for epiretinal membrane dissection during surgery and for the use of heavy liquids during membrane peeling.
\end{abstract}

The retina is capable of marked elastic stretch, and this may be seen in vivo in patients with diabetic traction retinal detachment ${ }^{1}$ and traction associated with retinopathy of prematurity or dominant exudative vitreoretinopathy. ${ }^{2}$ Retinal blood vessels become straightened, and the retina may have visible striae. Other in vitro work has demonstrated that the retina possesses elasticity. ${ }^{3,4.5}$ Zauberman and Berman measured the force required to separate retina from underlying retinal pigment epithelium. ${ }^{3}$ During this work they found that if free retina is pulled, it stretches until it tears. For cat retina, $3 \mathrm{~mm}$ strips tore at 300 $400 \mathrm{mg}$ force, and $5 \mathrm{~mm}$ strips at $450-600 \mathrm{mg}$. Retina is a thin membrane, approximately $0.1 \mathrm{~mm}$ thick for most locations, although its thickness increases to $0.23 \mathrm{~mm}$ in the peripheral macula. ${ }^{6}$ The retina is much thinner in the central fovea, but this area is very localised and is surrounded by thicker tissue. The retina is therefore a thin

From * Moorfields Eye Hospital, London EC1V 2PD. †Cavendish Laboratory, Cambridge CB3 OHE. ¥Manchester Royal Eye Hospital, Manchester M11.

Correspondence to J. D. Stevens, Moorfields Eye Hospital, City Road, London EC1V 2PD. membrane that has elastic properties and stretches before tearing occurs.

When an elastic membrane is stretched, the elastic limit is eventually reached and then the membrane tears partially, or completely, resulting in an irreversible deformation. ${ }^{7}$ In the latter case, the membrane does not, after stretching forces have been removed, return to its original shape or size since there has been disruption of the elastic structure.

What shape does the retina adopt when pulled? The shape is governed by:

(1) External forces acting in the retina.

(2) Elastic forces in the surface of the stretched membrane.

(3) Hydrostatic forces produced by the fluid in the eye in contact with the retina.

(4) Gravitational forces.

The resulting profile of deformation is difficult to model, because the stretching of the retina is not uniform as one moves from the base to the tip. The resulting stress will have two components, in a longitudinal direction and in hoops around the axis as shown (Fig. 1).

Tearing is likely to occur before the elastic limit is reached if there are 'weak spots' in the retina, such as areas of local thinning. The retina is not a homogeneous structure and the presence of blood vessels and breaks in the internal limiting membrane also alter local retinal strength. When an elastic membrane is under tension, any tear propagates, and the rate of propagation is greater as stress is increased.

\section{In vitro}

To investigate the properties of retina as an elastic membrane, fresh retina separated free of underlying retinal pigment epithelium, was taken and attached to an apparatus to apply a stretching force. If a strip of elastic tissue is taken and pulled, it tends to curl, forming a cylindrical shape. This is because pulling at the ends results in other tensions in the membrane. In addition to longitudinal 


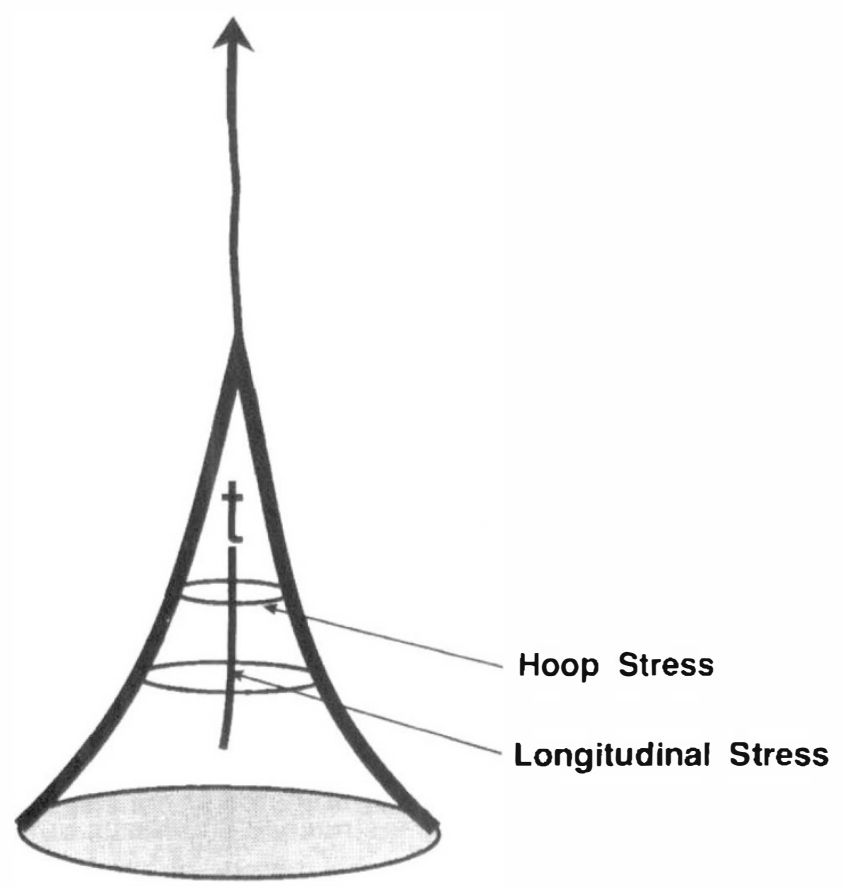

Fig. 1. Diagram of directions of tension within an elastic membrane.

stress in the direction of pull, hoop stresses perpendicular to the line of pull occur. The membrane becomes thinner, as described by Poisson's ratio, and tearing may occur at weak spots at the edges of a retinal strip, since it not a homogeneous material. To investigate the elastic properties of bovine retina, we took freshly enucleated eyes, and dissected out the retina. Retinal circular discs were taken which did not include the macula or the optic disc. This circular disc of retina could then be pulled at its centre, so eliminating the curling effect. From these experiments we have developed a mathematical model of the retina based upon the behaviour of retinal tissue observed in vitro. Pre-

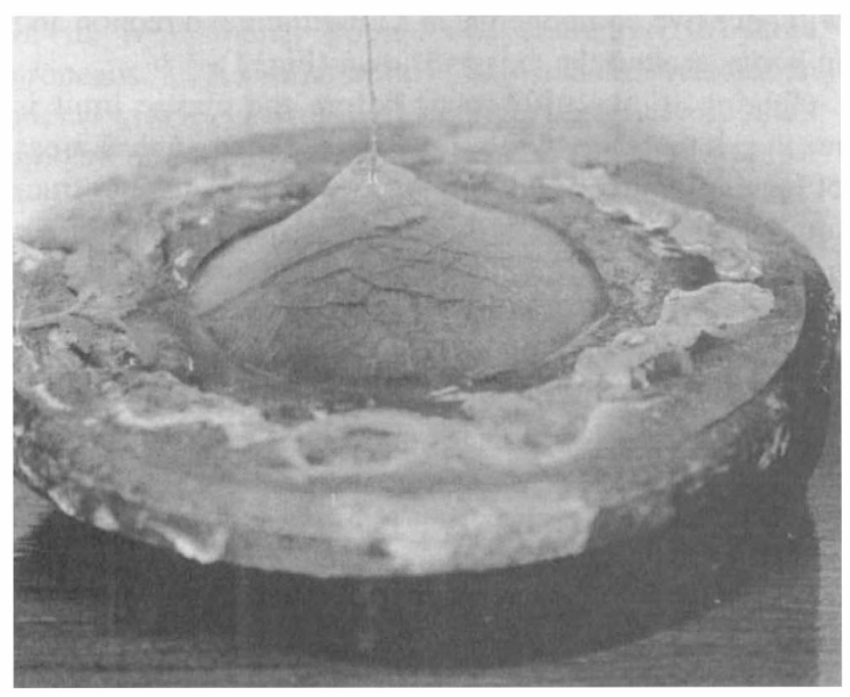

Fig. 2. Photograph of bovine retinal disc attached to a metal ring and tented up by a 10-0 nylon traction suture. Radial 'hoop' stress lines can be seen as well as vertical stress lines in the direction of the traction suture. dictions for local retinal strength in various situations can then be made, based upon the model.

\section{METHOD}

\section{In vitro}

Fresh bovine retinas were used for the study. Enucleation was performed minutes after slaughter, and the eyes were immediately frozen for transport. Retinas were dissected free anteriorly by $360^{\circ}$ retinotomy and incision around the optic disc. Free retina was then floated in Ringer's solution onto a metal disk of known internal diameter. This retina was then glued around the inner rim of the metal disk using Histoacryl glue. Discs of different internal diameters enabled varying surface areas of retina to be studied, thus allowing the retinal profile to be assessed for different retinal surface areas. All retinas were studied within hours of enucleation. A 10-0 nylon suture was suspended from a microbalance apparatus and a small application of butylacrylate (Histoacryl) glue run down the thread. Traction of known force could be applied to the suture. The retinal disc had surface moisture pipetted off the upper surface and the thread allowed to contact the centre of the retinal disc.

After a two minute period to allow the glue to bond, a gradual increase in traction was applied to the suture. The height of the apex of the disk was measured above the base line, and the force applied to the suture. A photographic apparatus was used to record the profiles of the stretched retinas (Fig. 2).

\section{RESULTS}

When a slowly increasing traction force was applied, over a period of two minutes, to the retinal discs, we noted that the retina became tented up, so increasing its surface area (Fig. 2). Elevation of the retina was proportional to the traction force applied (Table I). If the traction force was relaxed, the retina returned to its original base line (and surface area). It therefore behaved in the manner of an elastic membrane.

If the traction force was further increased, a point was reached (before tearing occurred) when the stretching of the retina became irreversible. If force was then removed

Table I. Elevation of retinal disc above base line

\begin{tabular}{ccccc}
\hline \multicolumn{2}{c}{$\begin{array}{c}\text { Ring diameter } \\
\text { Traction force }\end{array}$} & $\begin{array}{c}3.5 \mathrm{~mm} \\
\text { Elevation }\end{array}$ & & \multicolumn{2}{c}{$\begin{array}{c}\text { Ring diameter } \\
\text { Traction force }\end{array}$} & $\begin{array}{c}7.5 \mathrm{~mm} \\
\text { Elevation }\end{array}$ \\
\cline { 1 - 2 } \cline { 5 - 5 }$($ grams $)$ & $(\mathrm{mm})$ & & (grams) & $(\mathrm{mm})$ \\
\hline 0.0 & 0.0 & & 0.0 & 0.0 \\
0.25 & 0.5 & & 0.25 & 4.0 \\
0.50 & 3.0 & & 0.50 & 6.0 \\
0.75 & 3.5 & & 0.75 & 8.5 \\
1.00 & 4.0 & & 1.00 & 10.0 \\
1.25 & 4.5 & & 1.25 & 11.0 \\
1.50 & 3.0 & & 1.50 & 12.0 \\
1.75 & $($ tearing) & 1.75 & 13.0 \\
& & 2.00 & 13.0 \\
& & 2.25 & 13.5 \\
& & 2.50 & 13.5 \\
& & 2.75 & 13.5 \\
& & 3.00 & (tearing) \\
\hline
\end{tabular}




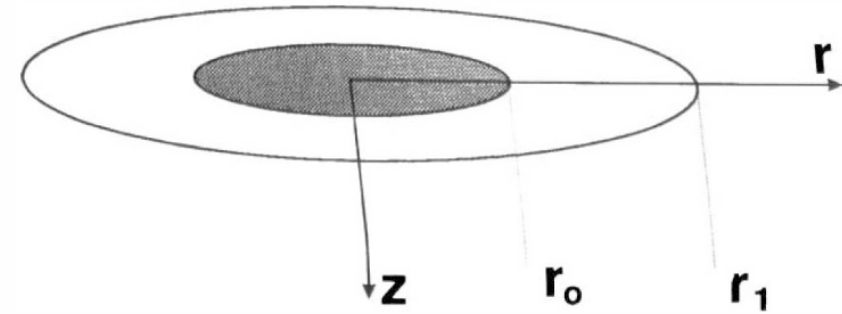

Fig. 3. Diagram of retinal disc with no traction, attached to peripheral ring and central fixed central area (representing glue attachment point).

from the traction suture, the retina sank down and was 'floppy', having a larger surface area than originally. At this phase of irreversible stretching, the retina altered its behaviour to a constant force. For a lesser force the retina would stretch a given amount and not stretch any further. At the phase of irreversible stretching, the retina continued to increase in surface area even though the same traction force remained applied. The elastic limit had been exceeded and the retina was displaying an irreversible increase in surface area as it continued to give way under the traction force. Tearing then occurred after a variable time of a few seconds to two minutes.

By altering the direction of pull of the traction suture, we found the shape of the tear altered. When force was applied at $90^{\circ}$ to the retinal surface, retinal tearing occurred at, or very near the edge of the glue spot. The tear in each instance was approximately circular. When the suture was applied at an acute angle, then tearing became oval in shape. This suggests that the elasticity of the retina is approximately isotropic, and there is no preferred direction of strength for the bovine peripheral retina. From these findings it appears justified to treat the retina as an isotropic elastic membrane.

\section{MATHEMATICAL MODELLING OF ELASTIC MEMBRANES}

We have modelled the stretching of the retina in the manner described above, by treating the retina as an elastic membrane with an isotropic elastic constant.

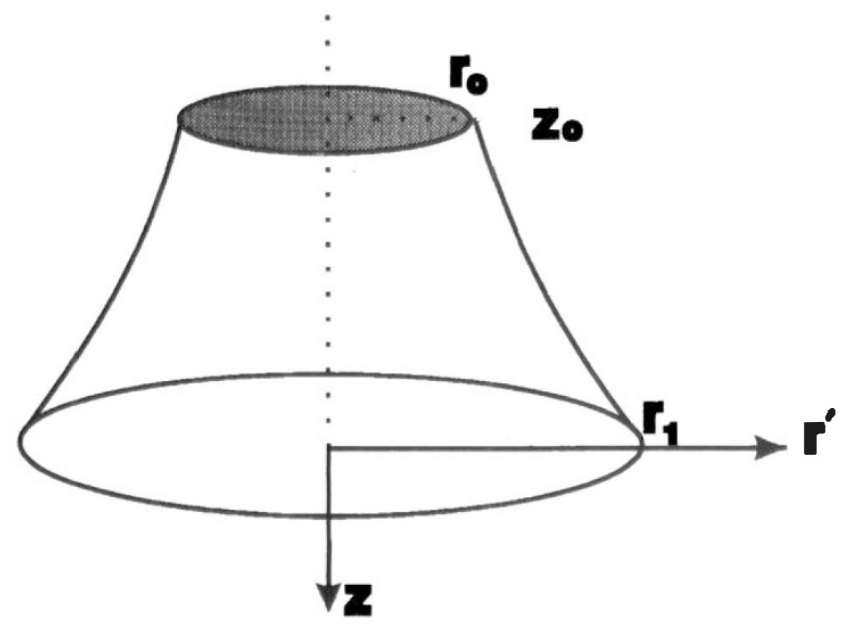

Fig. 4. Same retinal disc as Fig 3, but with traction applied in the $-Z$ direction.
Consider an elastic membrane, unstretched, and fixed at an outer radius $r_{1}$ and an inner radius $r_{0}$ (Fig. 3). Now apply $a$ force $f$ to the centre of the membrane (i.e. to the central fixed disc) in the $-z$ direction. The membrane will stretch and adopt a new position, given by $\left(r^{\prime}, z\right)$ (Fig. 4.). Where $r^{\prime}$ is given by: $r^{\prime}=u+r$ and where $u$ is the displacement. If an element was originally at coordinates $(r, 0)$, after stretching, that element's co-ordinates will be $\left(r^{\prime}, z\right)$. $u$ is the amount the element has moved in the radial direction. Clearly $u\left(r_{0}\right)=u\left(r_{1}\right)=0$ (Fig. 4.)

There are two parts to determining the shape of the membrane, i.e. $z\left(r^{\prime}\right), u\left(r^{\prime}\right)$.

\section{ELASTICITY}

Here we use the simple relationship stress $\propto$ strain, i.e. linear elasticity. There are two tensions (stresses) to consider; in the direction tangent to the membrane and coplanar with the $\mathrm{z}$ axis, and at the same point in the azimuthal direction (i.e. radial stress) (Fig. 5).

By considering the changes in lengths of elements in the surface, it can be shown that:

$$
\begin{aligned}
& t_{1}=k \quad\left(\frac{\left(1+\left(\frac{d z}{d r^{\prime}}\right)^{2}\right)^{1 / 2}}{1-\frac{d u}{d r^{\prime}}}-1\right. \\
& t_{\phi}=\frac{k u}{r^{\prime}-u}
\end{aligned}
$$

Where $\mathrm{k}$ is the elastic constant, and we have assumed it is the same constant for the 1 and $\phi$ directions. These elastic equations will be valid whether or not the membrane is in equilibrium. To complete the picture, we looked at the equilibrium conditions.

\section{EQUILIBRIUM}

After the force $f$ is applied, the membrane will initially

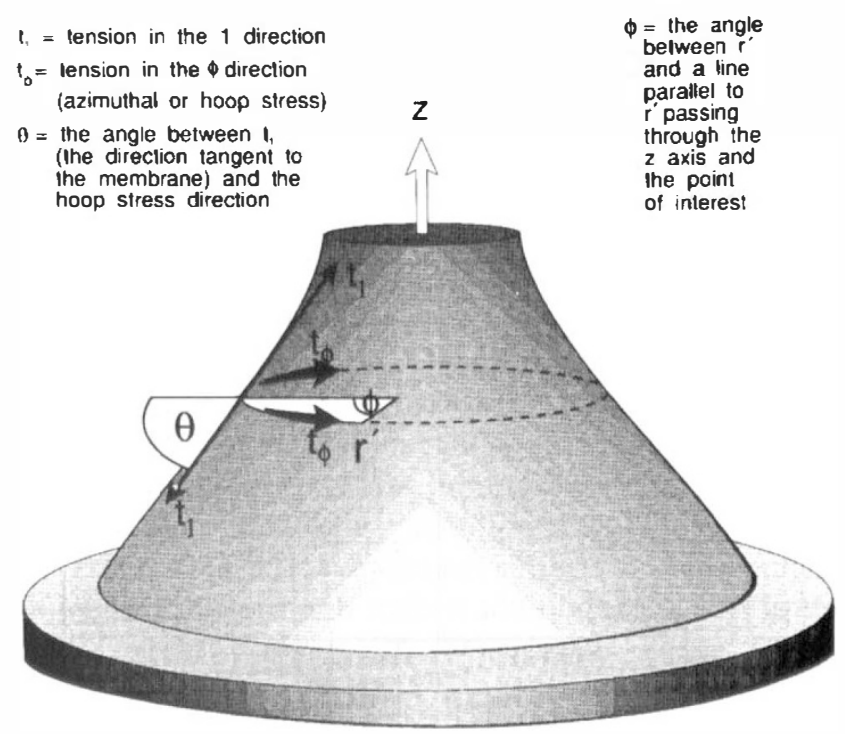

Fig. 5. Diagram of stresses within the stretched elastic membrane when the membrane is undergoing traction in the $-Z$ direction. 
move and come to rest with the base (at $r_{0}$ ) at a height of $z_{0}$. When the membrane is in equilibrium (i.e. at rest) the forces must balance, and by looking at the forces in the 1 and $\phi$ directions, it can be shown that:

$$
t_{1}=\frac{f}{2 \pi} \frac{1}{r^{\prime} \sin \theta}
$$

Now consider the same problem, but with a fluid density $\rho$ added above the membrane to a height $\mathrm{H}$, (Fig. $6)$. The elastic equations still hold, as they do not involve forces, but the equilibrium equations must be modified to take into account the changes in the applied forces. The equations now become:

\section{Fluid Of Density $P$ Added Above The Membrane}

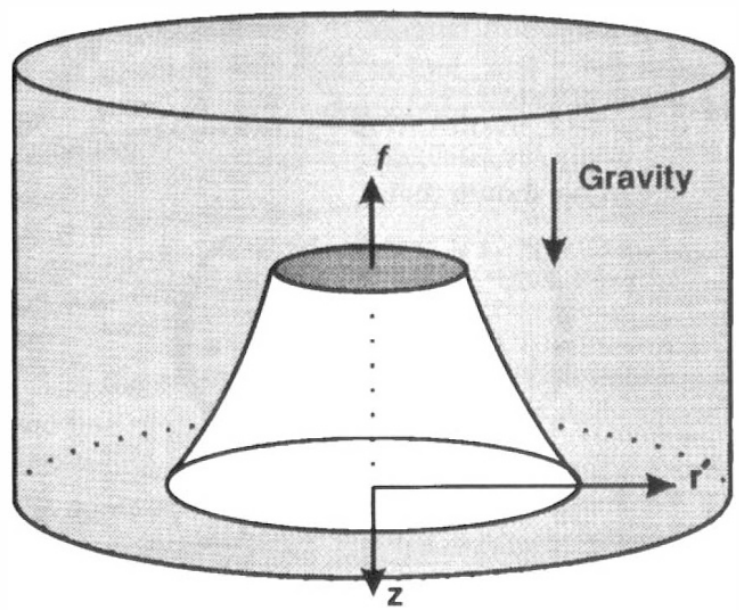

Fig. 6. The change in retinal profile after heavy liquid is added above an elastic membrane. The hydrostatic force exerts a pressure upon the membrane, altering its profile.

\section{Plot Of Height (z) Of Elastic Membrane versus Radial Coordinate ( $r$ )}

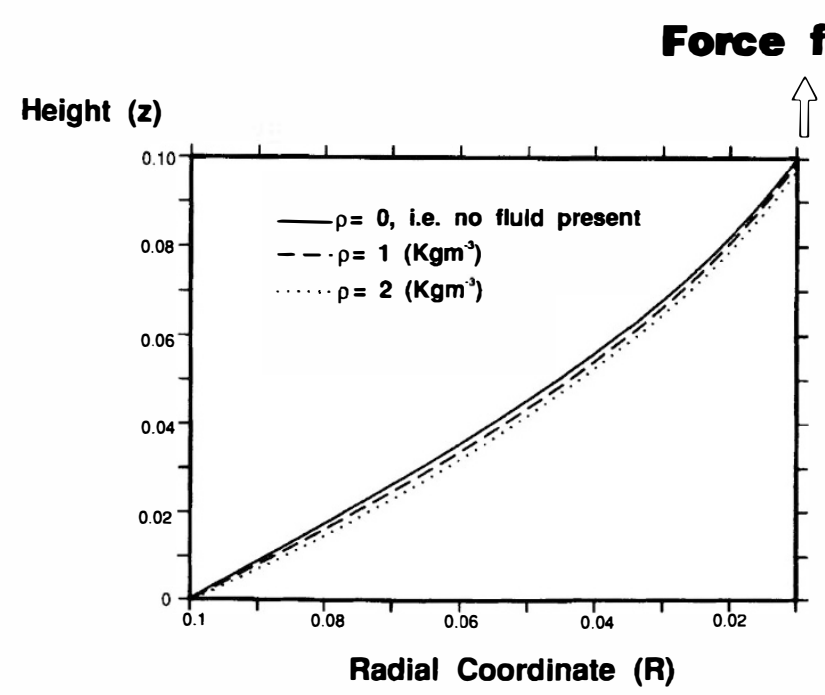

Fig. 7. Plot of height $(z)$ of elastic membrane against the radial coordinate $(r)$, for the 3 conditions where no added fluid is present, for fluid $\rho$ is $1 \mathrm{~kg} / \mathrm{m}^{-3}$ and for $\rho$ is $2 \mathrm{~kg} / \mathrm{m}^{-3}$.

$$
\text { where } \mathbf{F}_{\mathrm{k}}=2 \pi \mathrm{g} \int_{\mathrm{r}_{0}}^{\mathrm{t}_{1} 2 \pi \mathrm{r}^{\prime} \sin \theta=\mathrm{f}-\rho \mathbf{F}_{\mathrm{k}}}(\mathrm{z}(\alpha)-\mathrm{H}) \alpha \mathrm{d} \alpha
$$

$t_{\phi}=-\left(\frac{f / 2 \pi}{r^{\prime} \sin \theta}-\frac{\rho / 2 \pi}{r^{\prime} \sin \theta} F_{k}\right) \frac{d^{2} z}{d r^{2}} \frac{\cos ^{3} \theta}{\sin \theta} r^{\prime}-\rho \frac{(z-H) g}{\sin \theta} r^{\prime}$

The equations (1), (2), (3a) and (4a) can be re-arranged and re-written as three differential equations.

$$
\begin{aligned}
& \frac{\mathrm{dz}}{\mathrm{dr} \mathrm{r}^{\mathrm{N}}}=\mathrm{q} \\
& \frac{\mathrm{du}}{\mathrm{dr}}=1-\frac{\mathrm{k}_{1} \mathrm{r}^{\prime} \mathrm{q}\left(1+\mathrm{q}^{2}\right)^{1 / 2}}{\mathrm{k}_{1} \mathrm{r}^{\prime} \mathrm{q}+\left(1+\mathrm{q}^{2}\right)^{1 / 2}\left(1-\mathrm{k}_{2} \mathrm{~F}_{\mathrm{k}}\right)} \\
& \frac{d q}{d r^{\prime}}=\left(\frac{u}{r^{\prime}-u}+\frac{k_{2}}{k_{1}} 2 \pi g(z-h) \frac{r^{\prime}}{\left.\frac{\left(1+q^{2}\right.}{2}\right)^{1 / 2}}\right) \\
& \times\left(\frac{k_{1} q^{2}\left(1+q^{2}\right)^{1 / 2}}{k_{2} F_{k}-1}\right) \\
& \text { where } k_{1}=\frac{2 \pi k}{f} \text { and } k_{2}=\frac{p}{f}
\end{aligned}
$$

These equations have to be solved numerically. $f, r_{0}, r_{1}, k$ and $\mathrm{H}$ are fixed and the solutions $\mathrm{z}\left(\mathrm{r}^{\prime}\right), \mathrm{u}\left(\mathrm{r}^{\prime}\right)$ and $\mathrm{q}\left(\mathrm{r}^{\prime}\right)$ are found.

For example, in the graph of Height (z)/Radial co-ordinate (r) three curves are given (Fig 7.).

For all curves $r_{0}$ was fixed at 0.01 metres, $r_{1}$ at 0.1 metres, $f$ at 1 Newton, $\mathrm{k}$ at $10 \mathrm{~N} / \mathrm{m}$ and $\mathrm{H}$ at -0.5 metres.

The solid curve is for $\rho=0$ (i.e. no fluid present).

The dashed line is for $\rho=1 \mathrm{kgm}^{-3}$ (i.e. water above the membrane).

The dash-dot line is for $\rho=2 \mathrm{kgm}^{3}$ (i.e. liquid of twice the density of water above the membrane).

\section{Plot Of Tension In Tangent Direction versus Tension In Radial Coordinate}

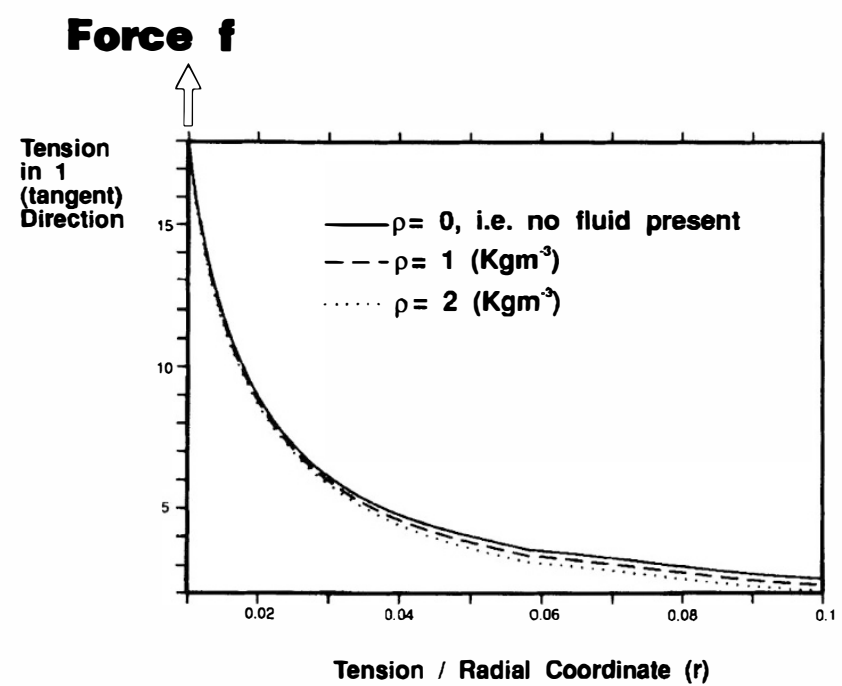

Fig. 8. Plot of tension, in the tangent direction, against the tension in the radial coordinate, for fluid $\rho$ is $1 \mathrm{~kg} / \mathrm{m}^{-3}$ and for $\rho$ is $2 \mathrm{~kg} / \mathrm{m}^{-3}$. In this example, there is much greater tension in the tangent direction. 


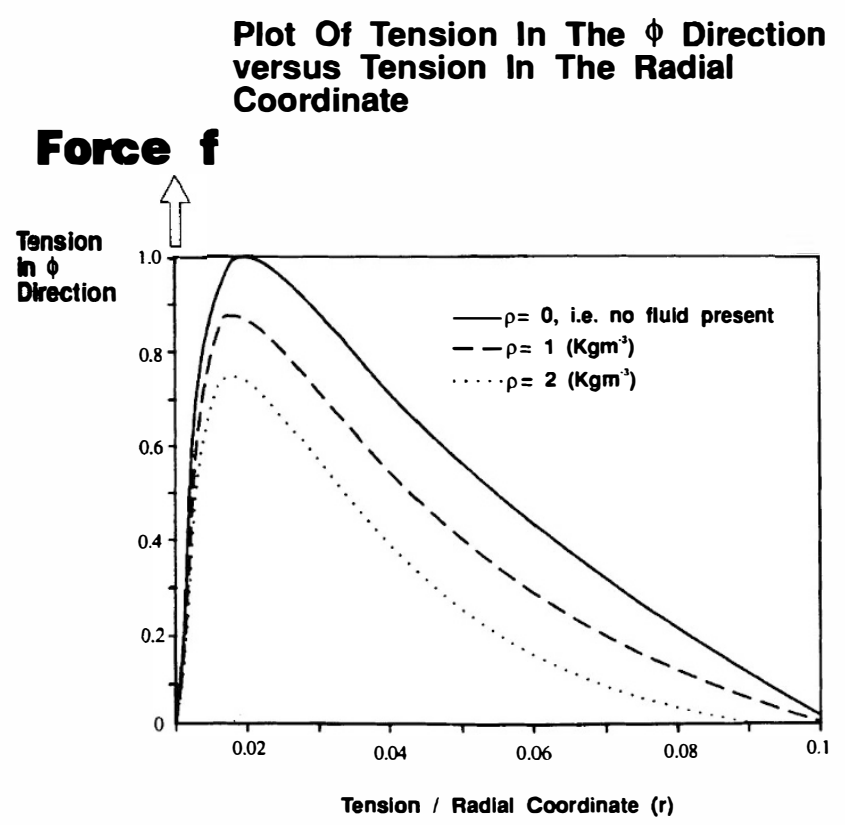

Fig. 9. Plot of tension in the $\rho$ direction, against the radial coordinate, for fluid $\rho$ is $1 \mathrm{~kg} / \mathrm{m}^{-3}$ and for $\rho$ is $2 \mathrm{Kg} / \mathrm{m}^{-3}$.

The graph of tension $\left(t_{1}\right) / r$ has the same three situations plotted, (Fig 8.) as does $t_{\phi} / r$ (Fig. 9).

These graphs demonstrate the change in retinal profile on adding fluid above the membrane.

They also demonstrate how the tension changes in each direction, as discussed below.

\section{OBSERVATIONS}

Consider an epiretinal membrane on the retinal surface, where retina is undergoing traction detachment. The retina is assumed, for this example, to remain fixed in position to the retinal pigment epithelium at the periphery of the detachment and only a portion is elevated by traction force from the epiretinal membrane. The retina therefore has internal tensions. In this situation we can model the use of heavy liquid within the posterior segment.

Height/r: Adding fluid "pushes" the retina back toward the base. Adding more or heavier fluid pushes the retina further back toward the base. This is what one might expect, i.e. adding fluid flattens out tented up retina.

Tensions: Both $t_{1}$ and $t_{\phi}$ are decreased, for most of the range, with fluid added and are futher decreased by adding more or heavier liquid.

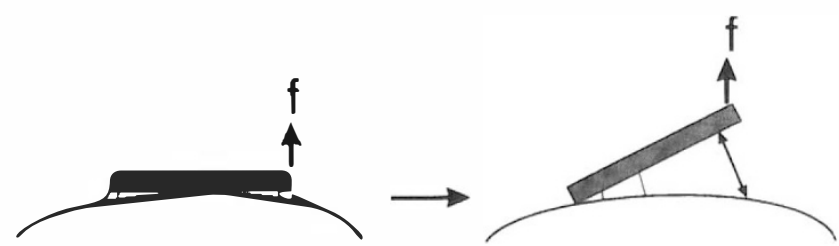

Fig. 10. Force f applied to a membrane required to separate the tip of a membrane from the retina. To achieve separation, the force on the bonds needs to be maximised, but with minimum tensions induced within the retina.
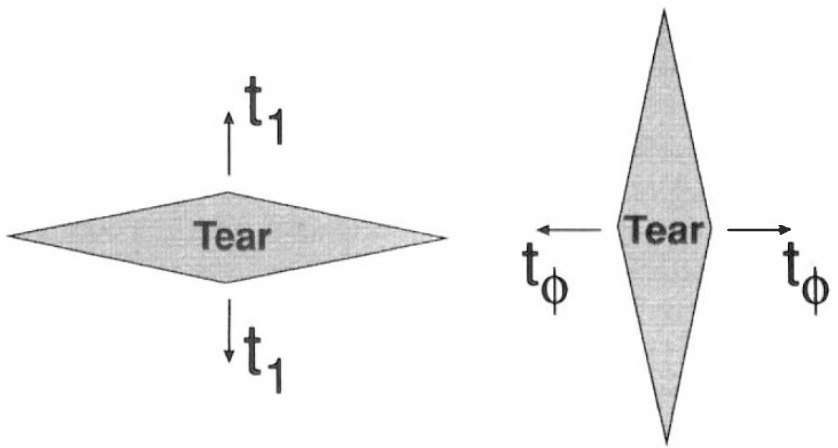

Fig. 11. Diagram illustrating tearing of the retina by forces in the $t_{l}$ and $t_{\phi}$ directions.

However note that the tensions in the 1 direction are approximately an order of magnitude greater than those in the $\phi$ direction, for the parameters we have chosen. It is likely that this is always the case within our model.

This example demonstrates that the probability of retinal tearing is decreased by adding fluid. Suppose that a given force $\mathrm{f}$ was necessary to produce at the tip a tension $\mathrm{t}$ that was just enough to separate a membrane from the retina (Fig. 10). By pulling on the membrane, tensions are introduced $\left(t_{1}\right.$ and $\left.t_{\phi}\right)$. If these tensions exceed the breaking point of the retina, a tear will occur and $t_{1}$, will produce tearing in the azimuthal direction (hoop stress) (Fig. 11), $\mathrm{t}_{\phi}$ will produce tearing in the longitudinal direction.

By adding fluid, $t_{\phi}$ is reduced to less than that required to produce tearing, but the tension at the tip still exceeds that required to produce separation of the membrane.

Here, we have assumed that the force required to remove the membrane remains the same. This may not be the same if the angle of attack changes. By angle of attack we refer to the angle between the retina and the edge of the epiretinal membrane. Empirically it is better to achieve a 'sharper' tip (Fig. 12).

Adding fluid can change the angle of attack, although in the example shown it does not appear to change by much. Separation is achieved by breaking bonds between membrane and retina. These can be stretched further by increasing the angle of attack. Empirically, less force is required to stretch the bonds to their breaking point. Separation is achieved by breaking bonds between membrane and retina by stretching them beyond their breaking points. At the same time, it is desirable to minimise the pulling force to reduce tensions in the membrane. A sharper angle of attack (a bigger angle between retina and attached membrane) results in longer bonds for the same pulling force. Here we are assuming the pull continues in the $\mathrm{z}$ direction $\left(90^{\circ}\right.$ to the retinal surface) because pulling in other directions will induce additional tensions in the

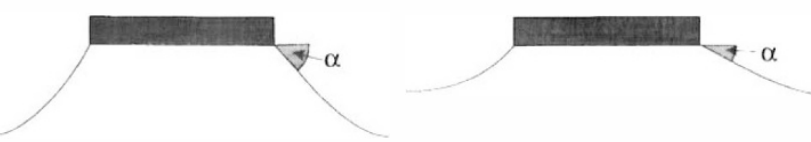

Fig. 12. Diagram illustrating the angle of attack between a membrane edge and the retina (angle $\alpha$ ). 
retina not considered here. Pulling at $180^{\circ}$ is the most advantageous to separate membrane from retina, but there is the danger that retina will tent up at the membraneretina separation point and induce large tensions within the retina. Under heavy liquid there is less tendency for retina to tent up, so we can anticipate a difference in the angle of attack. This situation has not been considered here. Although in the example shown it would be advantageous to add fluid and increase the density of the fluid, we have not shown that this would always be the case. The example given uses fictitious parameters and further work is required to render the model applicable to the human retina in vivo.

\section{DISCUSSION}

Bovine retina behaves like an elastic membrane when it is isolated and traction is applied to it in vitro. We have observed the behaviour of this tissue when stretched and developed a mathematical model of the retina as an elastic membrane. Modelling the retina in this way allows study of the use of heavy liquids and an optimum method for peeling an epiretinal membrane attached to the retina. We have shown that, when trying to separate an epiretinal membrane from an elastic membrane model of the retina, it may be advantageous to weigh down the elastic membrane with a heavy liquid. Too much heavy liquid however, can induce stresses in the membrane that are sufficient to cause tearing. It may be, therefore, that overfilling the eye with a heavy liquid causes retinal stretching, allowing only limited extra force to be applied before the retina tears. Thus there may be an increased risk of the retina tearing at a weak spot or region.

When peeling an epiretinal membrane that is relatively inelastic from an elastic membrane such as the retina, the optimum method of peeling is to pull the membrane back on itself to maximise the stretching force on the bonds between membrane and elastic membrane. In practice, the optimum peel angle is $90^{\circ}$, since this is the angle that max- imises the force vectors to the bonds between epiretinal membrane and retina without inducing excess force in the plane of the retina.

In the example we have taken, both the longitudinal stress and the azimuthal stress (hoop stress) are reduced by adding liquid, for the given pulling force $f$. This model demonstrates that, under certain specific circumstances, heavy liquids may decrease intra-retinal stress and reduce the risk of tear formation. Further work is required to apply the model to the in vivo situation, but our results provide a theoretical basis for the use of heavy liquids in separating epiretinal membranes from detached retina. We have shown it to be useful to think of the detached retina as an elastic membrane and to consider its properties as such. This work demonstrates that analytical modelling can be used to study the biophysical behaviour of retina under stress and the influence of heavy liquids. Future work should allow us to determine the densities and quantities of heavy liquids most appropriate for use in different configurations of retinal detachment.

Key words: elasticity, heavy, human, liquid, mathematics, modelling, perfluorocarbon, retina.

\section{REFERENCES}

1. Schepens CL: Retinal detachment. Saunders, 1983: 694-742.

2. Criswick VG and Schepens CL: Familial exudative vitreretinopathy. Am J Ophthalmol 1969, 68: 578-94.

3. Zauberman $\mathrm{H}$ and Berman ER: Measurement of adhesive forces between the sensory retina and the pigment epithelium. Expl Eye Res 1969, 8: 276-83.

4. Zauberman $\mathrm{H}$, de Guillebon $\mathrm{H}$ and Holly FJ: Retinal traction in vitro: biophysical aspects. Invest Ophthalmol 1972; 11: $46-55$.

5. de Guillebon $\mathrm{H}$ and Zauberman $\mathrm{H}$ : Experimental retinal detachment. Biophysical aspects of retinal peeling and stretching. Arch Ophthalmol 1972, 87: 545-8.

6. Michaels RG, Wilkinson CP and Rice TA: Retinal detachment. St Louis. CV Mosby, 1990: 19.

7. Flowers B and Mendoza E: Properties of matter. 1st ed. London. Wiley 1970: 50-53. 